\title{
Über Morphin-Chinolinäther
}

von

\author{
Dr. Paul Cohn.
}

Aus dem chemischen Laboratorium des Prof. Dr. Ed. Lippmann an der k. k. Universität in Wien.

(Vorgelegt in der Sitzung am 3. März 1898.)

Vor Kurzem habe ich in diesen Monatsheften ${ }^{1}$ eine Abhandlung veröffentlicht über die Darstellung und Eigenschaften von Chinolinäthern (wie 2-Chinolin-6-Oxychinolin und 2-Chinolin8'-Oxychinolin $\mathrm{C}_{9} \mathrm{H}_{6} \mathrm{~N}$.O. $\mathrm{C}_{9} \mathrm{H}_{6} \mathrm{~N}$ ), die durch Einwirkung von 2-Chlorchinolin auf die entsprechenden Oxychinoline erhalten wurden. Im Anschlusse an diese Mittheilung habe ich dieselbe Reaction auch bei Morphin versucht und bin dabei zu einem analog constituirten Körper gelangt.

Das Morphin $\mathrm{C}_{17} \mathrm{H}_{17}(\mathrm{OH})_{2} \mathrm{NO}$, das durch die Anwesenheit ron zwei Hydroxylgruppen sowie durch seine Löslichkeit in Alkalien hinreichend als Phenol charakterisirt erscheint, verhält sich jedoch dem 2-Chlorchinolin gegenüber weit träger als die vorgenannten Oxychinolinderivate. Erhitzt man Morphin im Ölbade selbst mit einem Überschusse von 2-Chlorchinolin, so findet keine Einwirkung statt, da die erstere Base nicht in Lösung gelangt und eine allmälig steigende Temperatur Verkohlung bewirkt. Die Anwendung von Lösungsmitteln war gleichfalls nicht von dem erwünschten Erfolge begleitet, da für das Morphin schwer ein das $P y^{\prime}-\alpha$-Chlorchinolin nicht zersetzendes, hochsiedendes (circa $266^{\circ}$ ) Mittel zu finden ist. Am besten operirt man in nachfolgender Weise.

1 Monatshefte für Chemie, Bd. XVII, S. 667 ff. und Sitzungsber. der kaiserl. Akad. der Wissensch. in Wien, mathem.-naturw. Classe, Bd. CV, Abth. II. b. 


\section{2-Chinolin-Morphin $\mathrm{C}_{17} \mathrm{H}_{17}\left(\mathrm{C}_{9} \mathrm{H}_{6} \mathrm{~N} . \mathrm{O}\right)(\mathrm{HO}) \mathrm{NO}$.}

$5-10 g$ 2-Chlorchinolin werden über freier Flamme bis nahe zum Sieden erhitzt (circa $250-260^{\circ}$ ) und in dasselbe in kleinen Portionen allmälig vollkommen wasserfreies (bei $120^{\circ}$ getrocknetes) Morphin eingetragen. Sofort beginnt eine Reaction, die man noch durch geringe Wärmezufuhr unterstützt, um mit dem weiteren Zusatz von Morphin fortfahren zu können, bis etwa $6-8 g$ eingetragen sind. Die ganze Operation soll behufs einer guten 'Ausbeute recht rasch verlaufen. Zum Schlusse erhitzt man noch unter fortwährendem Schütteln zum Sieden. Es ist dabei von der grössten Wichtigkeit, dass das Erhitzen sehr vorsichtig vorgenommen wird. Die Masse darf eine mässige Braunfärbung nicht überschreiten, wenn man ein brauchbares Product erhalten will. Es ist daher angezeigt, immer nur kleine Mengen in Angriff zu nehmen und die verschiedenen Reactionsproducte dann gemeinsam aufzuarbeiten. Nach dem Erkalten fügt man langsam unter gleichzeitigem Erwärmen am Wasserbade so lange verdünnte Salzsäure hinzu, bis Alles in Lösung gegangen ist. Diese wird noch mit der zwei- bis dreifachen Menge Wasser verdünnt und dann einige Stunden sich selbst überlassen. Man schüttelt hierauf die saure Lösung ein- bis zweimal mit etwas Äther aus, wobei etwa unverändertes 2-Chlorchinolin in die ätherische Lösung geht, um hieraus wieder gewonnen werden zu können. Die vom Äther abgelassene, schwach roth gefärbte Flüssigkeit wird sehr sorgsam mit ganz verdünnter Lauge partiell gefüllt und gelinde etwas erwärmt, während sich dabei durch kräftiges Schütteln die Gefässwände mit braunen harzartigen Theilen beschlagen. Sobald die Flüssigkeit nur mehr schwach lichtgelb gefärbt ist, wird rasch filtrirt und das Filtrat jetzt mit einem Überschuss von Lauge versetzt, wobei etwa noch unangegriffenes Morphin ${ }^{1}$ in Lösung geht, die neue Base vollständig ausgefällt und mit Äther extrahirt wird. Nach dem Trocknen und Abdestilliren des Äthers hinterbleibt eine schwach gelb gefärbte Lösung von

1 Fast immer bleibt ein Theil des Morphins unangegriffen, der durch Zusatz von Chlorammon zur alkalischen Lüsung fällt, die Bildung von Apomorphin wurde hierbei nicht beobachtet. 
syrupartiger Consistenz, die bei längerem Stehen krystallisirt. Man saugt ab, wäscht mit Äther nach und erhält so das neue Product von der Constitution:<smiles>C[14CH2][14C@H](C)N([14CH2]O)[14CH2]O</smiles>

Selten erscheint es noch nothwendig, die Substanz noch einmal aus Alkohol umzukrystallisiren. Die Base bildet kleine spitze Prismen, die sich häufig zu sternförmigen Krystallgruppen vereinigen oder grosse Warzen vorstellen, und schmilzt, im Capillarrohr erhitzt, bei $158^{\circ} \mathrm{zu}$ einer schwach gefärbten, hellen Flüssigkeit; in Wasser ist dieselbe nur wenig löslich (bläut rothes Lackmuspapier), leichter in kaltem Alkohol. Die Löslichkeit wird durch Wärmezufuhr wesentlich erhöht, desgleichen bei Äther, ${ }^{1}$ Benzol, Chloroform. Mineralsäuren nehmen gleichfalls leicht auf und bilden dann Salze, die sehr bitter schmecken. In Alkalien ist die Substanz, im Gegensatze zu Morphin, unlöslich, auch die für das Morphin so charakteristischen Reactionen mit Eisenchlorid (dunkelblaue Färbung) und Jodsäure etc. bleiben aus. Über Schwefelsäure getrocknet ist die Substanz wasser- und ätherfrei, und ergab die Analyse folgende Zahlen:

I. $0 \cdot 1551 g$ gaben, mit Kupferoxydasbest verbrannt, $0 \cdot 4315 \mathrm{~g}$ Kohlensäure und $0 \cdot 0876 \mathrm{~g}$ Wasser.

II. $0.1582 \mathrm{~g}$ gaben $0.4373 \mathrm{~g}$ Kohlensäure und $0.0874 \mathrm{~g}$ Wasser.

III. $0.2620 \mathrm{~g}$ gaben nach Dumas bei $24^{\circ} \mathrm{C}$. und $740 \mathrm{~mm}$ Barometerstand $17.0 \mathrm{~cm}^{3}$ feuchten Stickstoff. In 100 Theilen:

\begin{tabular}{|c|c|c|c|}
\hline \multicolumn{3}{|c|}{ Gefunden } & \multirow{2}{*}{$\begin{array}{l}\text { Berechnet für } \\
\mathrm{C}_{26} \mathrm{H}_{24} \mathrm{~N}_{2} \mathrm{O}_{3}\end{array}$} \\
\hline I. & II. & III. & \\
\hline C..... & $75 \cdot 39$ & - & $75 \cdot 72$ \\
\hline .. $6 \cdot 27$ & $6 \cdot 13$ & - & $5 \cdot 82$ \\
\hline$N \ldots \ldots-$ & - & $7 \cdot 07$ & $6 \cdot 79$ \\
\hline
\end{tabular}

1 Das frisch gefällte Hydrat ist amorph, ballt sich beim Erhitzen leicht zusammen und ist in Äther leichter löslich als die krystallisirte Substanz. 
Eine Löslichkeitsbestimmung der Substanz in absolutem Alkohol ergab, dass $20 \mathrm{~cm}^{3}$ bei $15^{\circ}$ gesättigter Lösung $0.7668 \mathrm{~g}$ bei $110^{\circ}$ getrocknet zurückliessen, oder 1 Theil löst sich in $20^{\circ} \cdot 1$ Theilen absoluten Alkohol bei $15^{\circ}$.

Die Base bildet neutrale und saure Salze, welch letztere fast durchwegs amorph sind und in Wasser zerfliessen. Das neutrale Chlorhydrat ist schwer zum Krystallisiren zu bringen; eine Lösung desselben bleibt beim Verdunsten selbst nach längerer Zeit als eine harte gallertartige Masse zurück, die auch durch Alkohol nicht gefällt wird. Mit Äther fällt ein weisser Niederschlag, der aber schon auf dem Filter zerfliesst.

\section{Chloroplatinat $\mathrm{C}_{26} \mathrm{H}_{24} \mathrm{~N}_{2} \mathrm{O}_{3} \cdot \mathrm{PtCl}_{6} \mathrm{H}_{2}$.}

Wird eine freie Salzsäure enthaltende Lösung mit Platinchlorid versetzt, so fällt ein blassgelb gefärbter Niederschlag, kleine undeutliche Krystalle bildend, der nach dem Abfiltriren auf der Platte getrocknet wird. Das Salz enthält Krystallwasser, das beim längeren Erhitzen auf $120^{\circ}$ entweicht und ungefähr 4 Moleküle beträgt. Beim Erhitzen im Capillarrohr schwärzt sich die Substanz zwischen $180-190^{\circ}$, ohne jedoch zu schmelzen.

$0 \cdot 3588, g$ der bis zur Gewichtsconstanz bei $120^{\circ}$ getrockneten

Substanz gaben geglüht $0 \cdot 0851 \mathrm{~g}$ Platin.

In 100 Theilen:

Pt ..... $\frac{\text { Gefunden }}{23 \cdot 71} \quad \frac{\begin{array}{c}\text { Berechnet für } \\ \mathrm{C}_{29} \mathrm{H}_{24} \mathrm{~N}_{2} \mathrm{O}_{3} \cdot \mathrm{PtCl}_{6} \mathrm{H}_{2}\end{array}}{23 \cdot 65}$

Neutrales Sulfat $\left(\mathrm{C}_{26} \mathrm{H}_{34} \mathrm{~N}_{2} \mathrm{O}_{3}\right)_{2} \mathrm{H}_{2} \mathrm{SO}_{4}+3 \mathrm{H}_{2} \mathrm{O}$.

Da dieses Salz sehr schön und leicht krystallisirt, so dient es am besten zur Charakterisirung der Base. Man erhält es durch genaues Neutralisiren derselben mit sehr verdünnter Schwefelsäure; nach kurzer Zeit oder beim mässigen Einengen erstarrt das Gemisch zu einem Brei, der aus feinen glänzenden Nädelchen besteht, die in kaltem Wasser schwer löslich sind, aus heissem Wasser schön krystallisiren und einige Ähnlichkeit mit dem neutralen Chininsulfat zeigen. Auf Zusatz von einigen Tropfen Salzsäure tritt sofort Lösung unter Bildung von saurem 
Salz ein. Von Alkohol wird das Salz schwer aufgenommen. Lufttrocken enthält es 3 Moleküle Krystallwasser, die bei $115^{\circ}$ entweichen. Im Capillarrohr erhitzt schmilzt es bei $257^{\circ}$ unter Aufbrausen zu einer braunen Flüssigkeit. Zur Löslichkeitsbestimmung wurden $20 \mathrm{~cm}^{3}$ einer bei $15^{\circ}$ gesättigten Lösung am Wasserbade eingeengt, hierauf bei $120^{\circ}$ getrocknet, es blieben $0.0487 \mathrm{~g}$ Substanz zurück oder 1 Theil löst sich in $410 \cdot 6$ Theilen Wasser von $15^{\circ}$.

$0.3580 \mathrm{~g}$ Substanz gaben bei $115^{\circ} 0.0210 \mathrm{~g} \mathrm{H} \mathrm{H}_{2} \mathrm{O}$.

In 100 Theilen:

$$
\mathrm{H}_{2} \mathrm{O} \ldots \underbrace{\text { Gefunden }}_{5 \cdot 86} \quad \underbrace{\left(\mathrm{C}_{20} \mathrm{H}_{24} \mathrm{~N}_{2} \mathrm{O}_{3}\right)_{2} \mathrm{H}_{2} \mathrm{SO}_{4}+3 \mathrm{H}_{2} \mathrm{O}}_{5 \cdot 53}
$$

$0.3150 \mathrm{~g}$ wasserfreier Substanz gaben, in verdünnter Salzsäure gelöst, mit Chlorbaryum gefällt u. s. w. $0.0780 \mathrm{~g}$ Baryumsulfat.

In 100 Theilen:

$$
\mathrm{H}_{2} \mathrm{SO}_{4} \ldots \underbrace{\text { Gefunden }}_{10 \cdot 41} \quad \frac{\begin{array}{c}
\text { Berechnet für } \\
\left(\mathrm{C}_{26} \mathrm{H}_{21} \mathrm{~N}_{2} \mathrm{O}_{3}\right)_{2} \mathrm{H}_{2} \mathrm{SO}_{4}
\end{array}}{10 \cdot 60}
$$

Chromat $\left(\mathrm{C}_{26} \mathrm{H}_{24} \mathrm{~N}_{2} \mathrm{O}_{3}\right)_{2} \mathrm{H}_{2} \mathrm{Cr}_{2} \mathrm{O}_{7}$.

Fügt man zu einer schwach angesäuerten Lösung der Base eine solche von Kaliumbichromat, so wird ein orange gefärbter Niederschlag gefällt. Derselbe wird nach dem Abfiltriren mit kaltem Wasser gut ausgewaschen, dann erst auf dem Thonteller und hierauf im Trockenkasten vorsichtig bei $100^{\circ}$ getrocknet. Beim stärkeren Erhitzen oder Kochen mit Wasser wird das Salz zerstört.

$0.2540 \mathrm{~g}$ Substanz gaben, bis zur Gewichtsconstanz geglüht, 0.0375 g Chromoxyd.

In 100 Theilen:

Berechnet für

$$
\mathrm{Cr}_{2} \mathrm{O}_{3} \ldots \underbrace{\text { Gefunden }}_{1+76} \quad \frac{\left(\mathrm{C}_{26} \mathrm{H}_{24} \mathrm{~N}_{2} \mathrm{O}_{3}\right)_{2} \mathrm{H}_{2} \mathrm{Cr}_{2} \mathrm{O}_{7}}{14.58}
$$




\section{Weinsaures Salz $\left(\mathrm{C}_{26} \mathrm{H}_{24} \mathrm{~N}_{2} \mathrm{O}_{3}\right)_{2} \mathrm{C}_{4} \mathrm{H}_{6} \mathrm{O}_{6}$.}

Man neutralisirt die Base mit verdünnter Weinsäurelösung. dampft am Wasserbade bis zur Trockne ein und lässt sodann einige Zeit über Schwefelsäure im Vacuum stehen, wobei eine harte, durchsichtige, amorphe Masse gebildet wird, welche mit absolutem Alkohol aufgenommen und in einem gut verschlossenen Gefässe stehen gelassen wird. Es bilden sich nach einiger Zeit an den Wänden sowie auf dem Boden des Gefässes prächtige, durchsichtige, glänzende, spitze Prismen, die sich auch zu Krystallgruppen formiren. Nach dem Abfiltriren und Waschen mit ein wenig absolutem Alkohol wird im Vacuum getrocknet. In Wasser, selbst verdünnten Weingeist ist das Salz sehr leicht löslich und daraus schwer zu krystallisiren. Bei $98^{\circ}$ schmilzt es unter Aufbrausen.

$0 \cdot 4760 \mathrm{~g}$ Substanz gaben, in Wasser gelöst, mit Chlorcalcium gefällt, filtrirt und geglüht, $0 \cdot 0502 \mathrm{~g}$ kohlensauren Kalk.

In 100 Theilen:

$\mathrm{CaCO}_{3} \ldots \underbrace{\text { Gefunden }}_{10 \cdot 55} \underbrace{\begin{array}{c}\text { Berechnet für } \\ \left(\mathrm{C}_{26} \mathrm{H}_{24} \mathrm{~N}_{2} \mathrm{O}_{3}\right)_{2} \mathrm{C}_{4} \mathrm{H}_{6} \mathrm{O}_{6}\end{array}}_{10 \cdot 26}$

\section{Pikrat $\left(\mathrm{C}_{26} \mathrm{H}_{24} \mathrm{~N}_{2} \mathrm{O}_{3}\right) \mathrm{C}_{6} \mathrm{H}_{2}\left(\mathrm{NO}_{2}\right)_{3} \mathrm{OH}$.}

Man setzt einer alkoholischen Lösung der Base in der Wärme eine gleichfalls alkoholische Pikrinsäurelösung zu. Anfangs zeigt der Kolbeninhalt flüssige Consistenz, beim Erkalten bilden sich aber kleine schön gelbe Krystalle, die in kaltem Alkohol schwer löslich sind. Im Capillarrohr erhitzt bräunt sich das Salz bei $230^{\circ}$ und schmilzt unter stürmischem Aufbrausen zwischen $250-252^{\circ} \mathrm{zu}$ einer theerigen Masse.

$0.2898 \mathrm{~g}$ Substanz gaben $0.6321 \mathrm{~g}$ Kohlensäure und $0.1156 \mathrm{~g}$ Wasser.

In 100 Theilen:
C . . . . $\frac{\text { Gefunden }}{39} \cdot \frac{-38}{38}$
$\mathrm{H} \ldots . .4 \cdot 43$

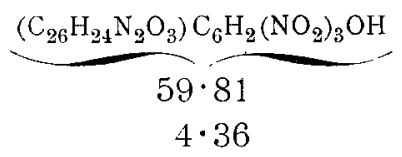

Berechnet für 
Gegen andere Agentien verhält sich das Morphinchinolin dem Morphin ähnlich, so entsteht mit Brom Substitution, mit Jod ein Perjodid u. s. w.

Versucht man im Morphinmolekül den Wasserstoff der zweiten Hydroxylgruppe durch einen zweiten Chinolinrest zu ersetzen, so gelingt dies nicht. Ein ähnliches Verhalten zeigt das erwähnte Alkaloid auch anderen Mitteln gegenüber wie Alkalien u. s. w. Unterwirft man das analog constituirte Codein, wo nur eine Hydroxylgruppe durch Methoxyl vertreten ist, derselben Reaction, so bleibt dasselbe auch beim längeren Erhitzen unverändert, eine Thatsache, die ebenfalls für das verschiedene Verhalten der Hydroxylgruppen im Morphin spricht und woraus deutlich hervorgeht, dass jener Wasserstoff, welcher im Codein durch die Methylgruppe substituirt ist, hier in Reaction getreten ist.

\section{Physiologisches Verhalten.}

Herr Dr. Alois Kreidl, Assistent am physiologischen Institute des Herrn Prof. Exner, war so freundlich, das neutrale Sulfat der Base auf seine physiologische Wirksamkeit zu untersuchen, und theilt mir hierüber Folgendes mit: Das Präparat erweist sich als ein starkes krampferzeugendes Gift, welches beim Kaltblüter schon in Dosen von $0.001-0.002 \mathrm{~g}$ letal wirkt. Es erzeugt dabei gleich zu Beginn heftige Krämpfe in allen Körpermuskeln besonders aber in den Respirationswegen (krampfhafter Verschluss der Glottis). Nach längerer Einwirkung tritt vollständige Lähmung bei erhaltener Sensibilität ein, das Herz schlägt weiter fort, während die Athmung sistirt ist. In diesem Stadium der vollständigen Lähmung zeigt das Thier eine Übererregbarkeit seines Nervensystems, indem es auf leichte Berührung auch auf Schallreize schon Krämpfe bekommt. Beim Warmblüter (Huud) ist die Dosis von $0.2 g$ bereits tödtlich. Auch das warmblütige Thier geht bei dieser Dosis unter gleichzeitigen heftigen Krampferscheinungen im Gebiete der gesammten Körpermuskulatur, des Zwerchfelles, des Kehlkopfes zugrunde. Der Angriffspunkt des Giftes ist wahrscheinlich das verlängerte Mark; geköpfte oder grosshirnlose Frösche zeigen bei subcutaner Injection das gleiche Bild. Auf das 
periphere Nervensystem der Kaltblüter ist das Gift ohne Einwirkung; die elektrische Erregbarkeit bleibt unverändert gleich den unversehrten Nerven.

Das genannte Gift setzt den Blutdruck bedeutend herab; schon die intravenöse Injection von $0.01 \mathrm{~g}$ bewirkt am Warmblüter eine bedeutende Blutdrucksenkung; in grösseren Dosen tritt neben dieser noch eine deutliche Pulsverlangsamung auf. Bei circa $0.12 \mathrm{~g}$ intravenös injicirt, was nahezu die letzte Dosis ist, sinkt der Blutdruck auf nahezu Null, während das Herz weiter schlägt. Es ist auffallend, dass die gleiche Dosis Morph. hydrochloric. sowie einer Auflösung von Chinolin in Acid. mur. ganz andere Erscheinungen bieten wie die des genannten Giftes.

\section{Optisches Verhalten.}

Herr Prof. Julius Mauthner hatte die Güte das Drehungsrermögen des Morphin-Chinolinäther-Sulfats zu untersuchen. Zur Bestimmung diente ein Landolt-Lippich'scher Apparat mit dreitheiligem Polarisator (Berl. Ber. XXVIII, 3, 3102) bei Natriumlicht mit Bichromat-Strahlenfilter. Die benützte Lösung enthielt auf $100 \mathrm{~cm}^{3} 3.0845 \mathrm{~g}$ Substan $z$ und $0.7 \mathrm{~g}$ Salzsäure. Sie zeigte im 1-Decimeterrohr eine Ablenkung von $\alpha=-2 \cdot 05^{\circ}$. Daraus ergibt sich ein spec. Drehungsvermögen $[\alpha]_{D}=-66 \cdot 46^{\circ}$. 